\title{
Case report: Neurosarcoidosis with hydrocephalus as a first presenting sign.
}

\author{
Valérie Schuermans ${ }^{1}$, Esther Yeung ${ }^{1}$, Wouter Henneman ${ }^{1}$, Linda Ackermans ${ }^{1}$, Roel \\ Heijboer $^{2}$, Rémy Mostard ${ }^{2}$, Winand Vos${ }^{2}$, Raphaël Pasmans ${ }^{2}$, and Olaf Schijns ${ }^{1}$ \\ ${ }^{1}$ Maastricht University Medical Centre+ \\ ${ }^{2}$ Zuyderland Medical Centre Heerlen
}

November 19, 2020

\begin{abstract}
This is a unique case describing hydrocephalus to be the primary presenting symptom of neurosarcoidosis. A 49-year-old woman with previous ischemic cerebral events presented with a gait disorder, urinary incontinence and cognitive impairment. Imaging showed obstructive hydrocephalus, progressive white matter abnormalities and basal leptomeningeal enhancement. Further diagnostics suggested sarcoidosis.
\end{abstract}

\section{Neurosarcoidosis with hydrocephalus as a first presenting sign; case report.}

Valérie N.E. Schuermans $\mathrm{MD}^{1,2}$, Esther Yeung $\mathrm{MSc}^{1,2}$, Wouter J.P. Henneman, MD, $\mathrm{PhD}^{3}$, Linda Ackermans $\mathrm{MD}, \mathrm{PhD}^{1,2}$, Roel Heijboer MD, $\mathrm{PhD}^{4}$, Rémy L.M. Mostard MD, $\mathrm{PhD}^{5}$, Winand Vos MD, $\mathrm{PhD}^{6}$, Raphaël Pasmans $\mathrm{MD}^{7}$, Olaf E.M.G. Schijns $\mathrm{MD}, \mathrm{PhD}^{1,2,8}$

Affiliations:

${ }^{1}$ Dept of. Neurosurgery, Zuyderland Medisch Centrum, Heerlen, The Netherlands

${ }^{2}$ Dept of. Neurosurgery, Maastricht University Medical Centre, Maastricht, The Netherlands

${ }^{3}$ Dept of. Radiology, Maastricht University Medical Centre, Maastricht, The Netherlands

${ }^{4}$ Dept of. Radiology and Nuclear Medicine, Zuyderland Medisch Centrum, Heerlen, The Netherlands

${ }^{5}$ Dept of. Pulmonology, Zuyderland Medisch Centrum, Heerlen, The Netherlands

${ }^{6}$ Dept of. Pathology, Zuyderland Medisch Centrum, Heerlen, The Netherlands

${ }^{7}$ Dept of. Neurology, Zuyderland Medisch Centrum, Heerlen, The Netherlands

${ }^{8}$ School for Mental Health and Neuroscience, Maastricht University, Maastricht, The Netherlands

Corresponding Author's name and current institution:

Valérie N.E. Schuermans MD ${ }^{1,2}$

Maastricht University Medical Centre, P. Debyelaan 25, 6229 HX Maastricht, The Netherlands

Corresponding Author's Email: valerie.schuermans@mumc.nl

Abstract word count: 49

Text word count: 1507 
Number of references: 19

Number of tables and/or figures: 4

Number of videos: 0

Keywords: Hydrocephalus, Neurosarcoidosis, Sarcoidosis, Ischemic Cerebral Events, Ventriculoperitoneal shunting

\title{
Key Clinical Message:
}

There is a possible relationship with cerebral ischemic events and neurosarcoidosis. It should be considered in the differential diagnosis in a case of unexplained hydrocephalus, vascular white matter lesions and vasculitis related findings.

\begin{abstract}
This is a unique case describing hydrocephalus to be the primary presenting symptom of neurosarcoidosis. A 49-year-old woman with previous ischemic cerebral events presented with a gait disorder, urinary incontinence and cognitive impairment. Imaging showed obstructive hydrocephalus, progressive white matter abnormalities and basal leptomeningeal enhancement. Further diagnostics suggested sarcoidosis.
\end{abstract}

\section{Introduction}

Sarcoidosis is a chronic, granulomatous disease of unknown etiology ${ }^{1,2}$ which mainly affects the respiratory and lymphatic systems. Rarely ( $5-13 \%$ of sarcoidosis patients), the central nervous system (CNS) is involved ${ }^{3}$, or is the only affected system $(1 \%)^{4}$. Although, post-mortem studies suggest that ante-mortem diagnosis is only made in $50 \%$ of patients with sarcoidosis with nervous-system involvement ${ }^{5}$. The first report of neurosarcoidosis was published in $1905^{6,7}$. Cranial nerve deficits are the most common clinical presentation, particularly a facial nerve palsy. Other symptoms include endocrine dysfunction, seizures, encephalopathy, peripheral neuropathy, meningitis, spinal cord dysfunction or myopathy ${ }^{8}$. Neurosarcoidosis can present with granulomas at the surface of the brain, preferably at the skullbase, with a perivascular distribution with parenchymal involvement, or even as a tumefactive mass ${ }^{9}$.

Contrast-enhanced MRI is the preferred examination when a granulomatous disease is suspected ${ }^{10,11}$, and can be used to monitor the course of the disease. A final diagnosis of sarcoidosis requires histological confirmation of a noncaseating granuloma in radiologically involved tissue ${ }^{9}$. The differential diagnosis however is extensive due to the variation in presenting symptoms and affected organs, other pathologies thus need to be ruled out.

We describe a unique case with symptomatic hydrocephalus as the first sign of sarcoidosis. The patient's recent history of ischemic cerebrovascular disease could possibly be attributed to the previous undetected sarcoidosis.

\section{Case presentation}

A 49-year-old woman was admitted to the neurology department of a teaching hospital with progressive complaints of gait disturbance, urinary incontinence, apathy, bradyphrenia and short-term memory impairment. Within 5-6 weeks she became unable to walk. Her medical history revealed hypertension, dyslipidemia, transient ischemic attacks (TIA's) and a pontine stroke three years earlier. She never fully recovered from her pontine stroke with persistent complaints of cognitive impairment, fatigue and some difficulty walking. Computed tomography (CT) was performed (fig 1 matter lesions and suspicion of vasculitis related findings) showing quadriventricular hydrocephalus compared to imaging one year earlier, as well as confluent white matter changes, most notably in the deep white matter of both frontal lobes. Repeated lumbar punctures with normal opening pressures (10-13 $\mathrm{mmHg}$ ) temporarily improved her clinical condition, which was objectified with a gait analysis and the cognitive complaints and incontinence improved subjectively as well. The cerebral spinal fluid (CSF) analysis showed a mild lymphocytic pleocytosis $\left(16^{*} 10^{\mathrm{E}} 6 / 1\right)$, with an erythrocyte count of 0 , normal glucose $(2,2 \mathrm{mmol} / \mathrm{l})$, slightly elevated proteins $(0,94 \mathrm{~g} / \mathrm{l})$, albumin $(655 \mathrm{mg} / \mathrm{l})$ and $\operatorname{IgG}$ 
(98 mg/l), intrathecal igG was $0 \mathrm{mg} / \mathrm{ml}$, with $5 \mathrm{IgG}$ oligoclonal bands in CSF and 2 in serum. Cell count showed $0 \%$ polymorphonuclear cells and $100 \%$ mononuclear cells. Infectious and immunologic screening in CSF was negative.

Magnetic resonance imaging (MRI) of the brain confirmed the hydrocephalus and frontal white matter changes, but also showed progressive white matter changes in the other lobes, basal nuclei and cerebellum compared to earlier imaging. On sagittal imaging, CSF pulsation artifacts could be appreciated in the Sylvian aqueduct, but not in the foramen magnum or around the median or lateral apertures of the fourth ventricle. Together with dilatation of the fourth ventricle and 'ballooning' of both lateral apertures, this suggested an obstruction of the fourth ventricle CSF-outflow (fig 2). Contrast-enhanced MRI was performed later, showing linear and patchy enhancement within the posterior cranial fossa and basal regions of the brain (fig 3 ). The patient was referred to the neurosurgery department for ventriculoperitoneal shunt (VPS) placement. Intracranial pressure appeared to be slightly elevated during surgery, although not measured.

Analysis of peroperative obtained CSF showed no malignant cells; a fluorescence-activated cell sorting (FACS) -analysis could not be performed due to the low cell-count. Due to the differential diagnosis of leptomeningeal metastases, basal leptomeningitis (including granulomatous disease), vasculitis, lymphoma, auto-immune or paraneoplastic pathologies, a PET-CT was performed. The PET-CT showed supraclavicular, mediastinal and parailliacal lymphadenopathy. An ultrasound-guided puncture was performed in the supraclavicular lymph node. Histological examination showed a granulomatous disease with a strong preference for sarcoidosis (fig 4). This was confirmed by a second puncture of one of the PET-positive lymph nodes. Both a Mantoux test and a PCR for Mycobacterium tuberculosis proved negative and tuberculosis was thus excluded.

\section{Discussion}

Sarcoidosis is a multi-systemic disease, characterized by cellular immunity activity with formation of noncaseating granuloma in various organ systems. Diagnosis of sarcoidosis is often a complex, time-consuming trajectory due to the variety of clinical presentations and the fact that laboratory blood and CSF examination is frequently unspecific. As seen in our patient; mononuclear inflammatory cells, elevated protein and occasionally low glucose. Three criteria are required to diagnose sarcoidosis: clinical and matching radiologic manifestations, a histological prove of a noncaseating granuloma and the exclusion of alternative diseases. The Neurosarcoidosis Consortium Consensus Group has developed a diagnostic criterion for neurosarcoidosis ${ }^{3,9}$. Cranial and peripheral neuropathies are the major presenting symptoms, together with myopathy, seizures, gait disorders and cognitive decline. In 1-7 \% the vestibulocochlear nerve is affected ${ }^{12,13}$. In the present case, involvement of VIIIth cranial nerve could be suspected due to her unsteadiness with walking.

Neurosarcoidosis in the basal leptomeninges appears as thickening and enhancement (either focal, multifocal or diffuse) on gadolinium-enhanced T1-weighted MRI. It is usually indistinguishable from the pattern seen in tuberculous meningitis or CNS lymphoma ${ }^{8}$, which both may have a similar clinical presentation. Therefore, a histological diagnosis of the radiological aberrant tissue is necessary. In tuberculous meningitis, the basal leptomeningeal involvement is known to be due to extension and/ or rupture of a Rich focus (subpial or subependymal focus) to the subarachnoid spaces and ventricular system, which causes enhancement of the exudate $^{14}$. A similar mechanism in neurosarcoidosis has not been described.

Parenchymal involvement often follows a perivascular spread, with T2/FLAIR hyperintense white matter changes and / or perivascular enhancement ${ }^{15}$. The progressive T2 hyperintensities in the deep white matter, observed in our patient, should be interpreted as parenchymal involvement.

The question remains whether the ischemic strokes and progressive vascular white matter changes in our patient, as a consequence of small-vessel disease, can be attributed to neurosarcoidosis. Although rarely, it has been reported that neurosarcoidosis can cause arterial and venous ischemic and hemorrhagic strokes or TIA's due to granulomatous inflammation of intracranial blood vessels ${ }^{15}$. This has mainly been described in medium and small vessels, literature of large vessel involvement is scarce and is thought to be due to compression by granuloma's rather than granulomatous inflammation or vasculitis ${ }^{1516}$. 
There is still some uncertainty about the underlying pathophysiological mechanism of stroke-like symptoms in patients with sarcoidosis. A retrospective study evaluated patients with sarcoidosis-related strokes and concluded that a stroke was the first presenting symptom in $40 \%$ of the patients ${ }^{16}$. In about $92 \%$ of neurosarcoidosis patients presenting with stroke, sarcoidosis appeared to be present in other non-nervous systems after additional examination. The authors suggest that the granulomatous invasion of the blood vessel walls is the mechanism behind sarcoidosis related cerebrovascular events rather than sarcoidosis related vasculitis. It is thought that vasculitis due to sarcoidosis is more commonly resulting in a slowly progressive encephalopathy than an ischemic stroke ${ }^{17}$. A mortality rate of $23 \%$ is reported in sarcoidosis patients presenting with stroke ${ }^{16}$.

Due to the three-year time interval between stroke and diagnosis of neurosarcoidosis, it remains debatable whether this could have been a first warning sign of neurosarcoidosis in our patient.

Hydrocephalus is described to emerge in the course of neurosarcoidosis in 5 to $12 \%$ of affected individuals 4. Usually this is a communicating hydrocephalus as a consequence of a decrease in CSF resorption due to leptomeningeal involvement. Though, CSF outflow obstruction caused by involvement of the basal leptomeninges in the inflammatory process and consequent adhesions, loculations in the ventricular system or infiltration of the choroid plexus can also lead to hydrocephalus ${ }^{18,8}$.

Scarce literature is available on the treatment of neurosarcoidosis and is mainly based on expert opinion and small retrospective studies. Typically, initial treatment consists of corticosteroids, although the exact mechanism is still unknown. It is hypothesized that the benefit is due to the anti-inflammatory and immunemodulating effects ${ }^{11,19}$. The benefit of this treatment varies widely from substantial improvement to no improvement at all. As a second step, a steroid-sparing or second-line agent, like Methotrexate (MTX) or mycophenolate can be considered. When the need for immunosuppressive treatment is chronic, upfront start of combination therapy of both corticosteroids and a steroid-sparing or second-line agent can be preferred. Further treatment options include TNF-alpha inhibitors such as infliximab, although treatment is expensive and poses a higher risk for infections and other toxicities ${ }^{9,19}$.

\section{Conclusion}

The present unique case illustrates a rare presentation of sarcoidosis, with symptoms related to obstruction hydrocephalus. We discuss the possible relationship with a previous episode of multiple cerebral ischemic events and neurosarcoidosis. The causes of hydrocephalus are most frequently a combination of CSF obstruction and disturbed resorption mechanisms due to basal meningeal granulomatous and inflammatory adhesions. Neurosarcoidosis should be considered in the differential diagnosis in a case of unexplained hydrocephalus, vascular white matter lesions and suspicion of vasculitis related findings.

\section{Patient Consent}

The patient has consented to the submission of the case report for submission to the journal.

\section{Acknowledgements}

The authors are thankful to the patient for cooperating in the writing of this manuscript.

\section{Author Contribution:}

VS, EY: data collection and drafting of the manuscript

WH, RH: radiologic data collection, images and description, critically revising manuscript

WV: pathologic data collection, images and description

LA, RM, RP, OS: critically revising manuscript

\section{Conflict of interest:}

The authors declare that they have no competing interests. 


\section{References}

\#1 1. Iannuzzi MC, Rybicki BA, Teirstein AS. Sarcoidosis. N Engl J Med . 2007;357(21):2153-2165. doi:10.1056/NEJMra071714

2. Lower EE, Broderick JP, Brott TG, Baughman RP. Diagnosis and management of neurological sarcoidosis. Arch Intern Med . 1997;157(16):1864-1868.

3. Stern BJ, Krumholz A, Johns C, Scott P, Nissim J. Sarcoidosis and its neurological manifestations. Arch Neurol . 1985;42(9):909-917. doi:10.1001/archneur.1985.04060080095022

4. Smith JK, Matheus MG, Castillo M. Imaging manifestations of neurosarcoidosis. AJR Am J Roentgenol . 2004;182(2):289-295. doi:10.2214/ajr.182.2.1820289

5. Hoitsma E, Faber CG, Drent M, Sharma OP. Neurosarcoidosis: a clinical dilemma. Lancet Neurol . 2004;3(7):397-407. doi:10.1016/S1474-4422(04)00805-1

6. Burns TM. Neurosarcoidosis. Arch Neurol . 2003;60(8):1166-1168. doi:10.1001/archneur.60.8.1166

7. COLOVER J. SARCOIDOSIS WITH INVOLVEMENT OF THE NERVOUS SYSTEM.Brain . 1948;71(4):451-475. doi:10.1093/brain/71.4.451

8. Ginat DT, Dhillon G, Almast J. Magnetic resonance imaging of neurosarcoidosis. J Clin Imaging Sci . 2011;1:15. doi:10.4103/2156-7514.76693

9. Stern BJ, Royal W 3rd, Gelfand JM, et al. Definition and Consensus Diagnostic Criteria for Neurosarcoidosis: From the Neurosarcoidosis Consortium Consensus Group. JAMA Neurol . 2018;75(12):1546-1553. doi:10.1001/jamaneurol.2018.2295

10. Zajicek JP, Scolding NJ, Foster O, et al. Central nervous system sarcoidosis-diagnosis and management. QJM . 1999;92(2):103-117. doi:10.1093/qjmed/92.2.103

11. Lacomis D. Neurosarcoidosis. Curr Neuropharmacol . 2011;9(3):429-436. doi:10.2174/157015911796557975

12. Yacoub HA, Al-Qudah ZA SN. Cranial neuropathies in sarcoidosis. World J Ophthalmol . 2015;5(1):16-22.

13. Krumholz A, Stern BJ, Stern EG. Clinical implications of seizures in neurosarcoidosis. Arch Neurol . 1991;48(8):842-844. doi:10.1001/archneur.1991.00530200084023

14. S.E. Knapen, P. Goswani KMAV. Uw Diagnose? Tijdschr Neurol Neurochir . 2019;120(4):161-163.

15. Bathla G, Singh AK, Policeni B, Agarwal A, Case B. Imaging of neurosarcoidosis: common, uncommon, and rare. Clin Radiol . 2016;71(1):96-106. doi:10.1016/j.crad.2015.09.007

16. Jachiet V, Lhote R, Rufat $\mathrm{P}$, et al. Clinical, imaging, and histological presentations and outcomes of stroke related to sarcoidosis. J Neurol . 2018;265(10):2333-2341. doi:10.1007/s00415-018-9001-x

17. Brown MM, Thompson AJ, Wedzicha JA, Swash M. Sarcoidosis presenting with stroke. Stroke . 1989;20(3):400-405. doi:10.1161/01.str.20.3.400

18. Hesselmann V, Wedekind C, Terstegge K, et al. An isolated fourth ventricle in neurosarcoidosis: MRI findings. Eur Radiol . 2002;12 Suppl 3:S1-3. doi:10.1007/s00330-002-1369-3

19. Voortman M, Daan Fritz, O.J.M. Vogels, D. van de Beek, J. de Vries, Mieke C. Brouwer MD. Clinical manifestations of neurosarcoidosis in the Netherlands. J Neurol Neurosci . 2019;10(2):292.

Neurosarcoidosis with hydrocephalus as a first presenting sign; report of a unique case.

Legends of Tables and/or figures

Figure 1. 
Transverse CT images without contrast show triventricular hydrocephalus, with extensive white matter changes in both frontal lobes, external capsule and around the fourth ventricle.

\section{Figure 2.}

MRI. Transverse FLAIR (a) shows dilatation of both lateral ventricles and extensive white matter changes, in which the vascular white matter lesions coalesce with the periventricular fluid accumulation due to hydrocephalus. $1 \mathrm{~mm}$ maximum intensity projection (MIP) of high resolution T2 weighted sequence (b) illustrates the 'ballooning' of the lateral foramina of Luschka (arrows). Sagittal T2-weighted image (c) shows dilatation of the fourth ventricle, with a prominent flow artefact over the cerebral aquaduct $\left(^{*}\right)$. Arrow points to a lacune in the pons.

\section{Figure 3.}

Transverse (a) and sagittal (b) contrast enhanced T1 weighted images show a pattern of basal leptomeningeal enhancement in the posterior fossa and basal portions of the brain.

\section{Figure 4 .}

Histology of lymph node biopsy showing a noncaseating granuloma.
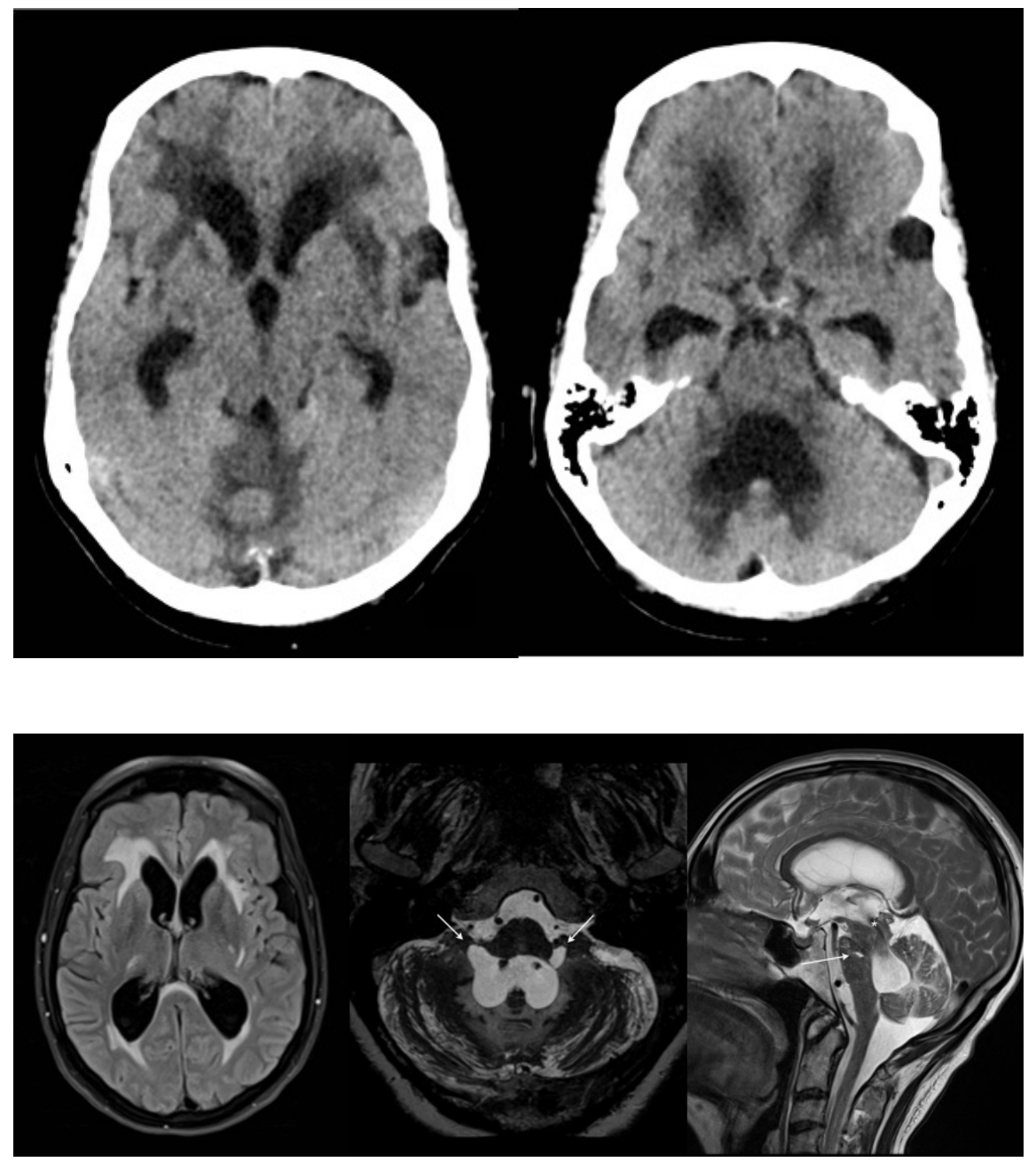

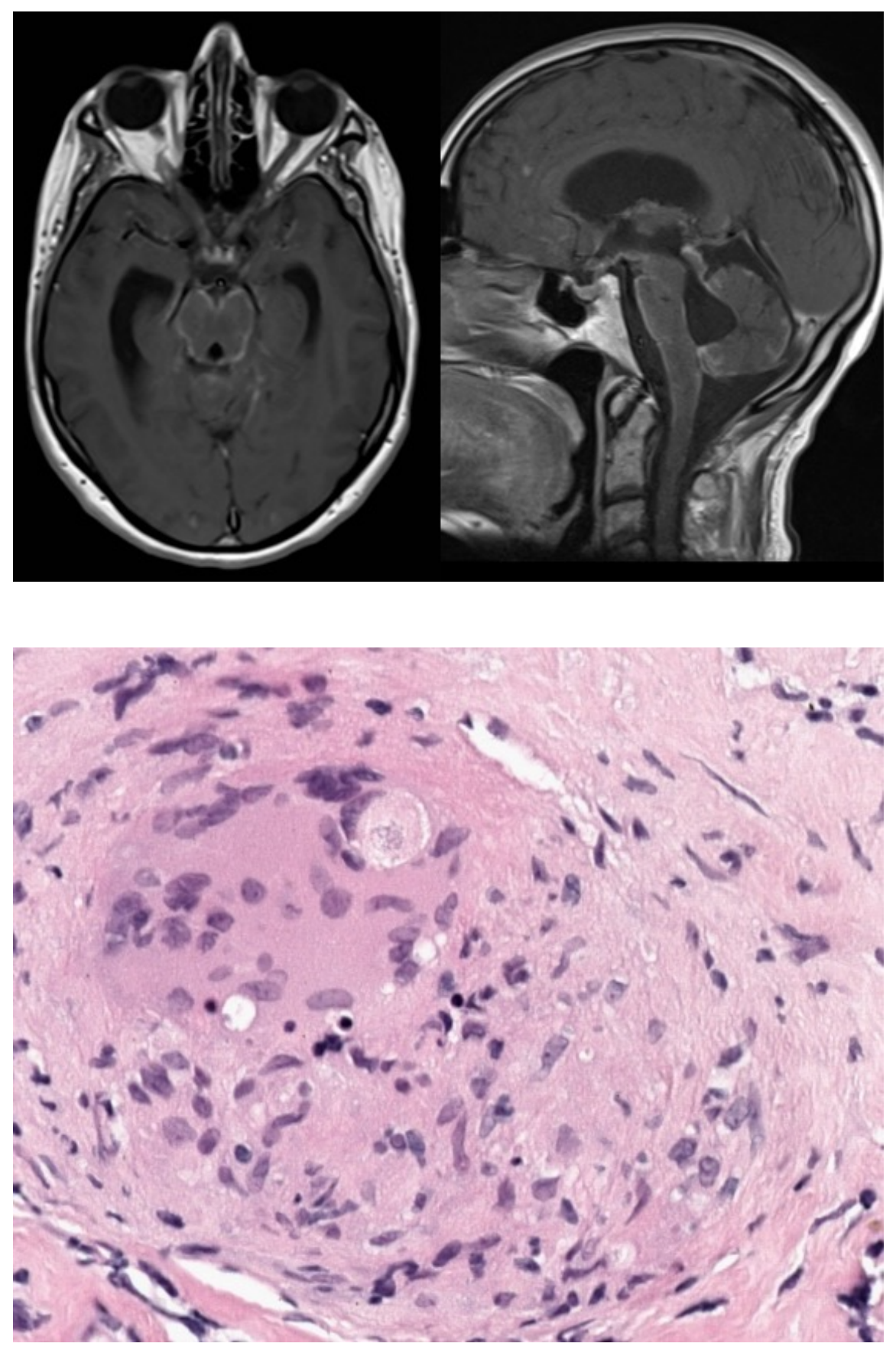